\title{
Effets de la réforme cotonnière en Espagne
}

\section{Manuel Arriaza \\ Humberto Capellán}

Instituto de Investigación y Formación Agraria y Pesquera (IFAPA)

Centro "Alameda del Obispo"

Apartado 3092

14080 Córdoba

Espagne

$<$ manuel.arriaza@juntadeandalucia.es>

<humberto.capellan@juntadeandalucia.es>

\section{Résumé}

L'article analyse les effets de la nouvelle réforme du coton (NRC) en Espagne jusqu'à la campagne 2007-2008, soit deux ans après son application mais avant son amendement en juin 2008. Il se distingue des travaux antérieurs par une meilleure mise en évidence des processus en ouvre dans la baisse de la production constatée. L'analyse est réalisée à partir des statistiques officielles tenues par la Province d'Andalousie et des informations recueillies au cours d'entretiens avec les acteurs du secteur coton. Les entretiens ont été conduits pour amener les producteurs à préciser les conséquences éventuelles de la NRC sur leurs pratiques culturales. Une telle approche affranchit de la nécessité de bâtir des modèles mathématiques très complexes pour identifier les impacts de la NRC sur la façon de cultiver le coton et sur la production obtenue. Alors que la production cotonnière était assez stable au cours de la période 1996-2005, l'application de la NRC en 2006 a contribué à réduire la superficie de plus de $30 \%$. La baisse de la production a été plus forte - autour de $60 \%$ dès la première année d'application - en raison d'un effondrement du rendement moyen lié à une forte réduction du recours aux intrants agricoles. Si une telle évolution est positive sur le plan environnemental, les pertes d'emplois industriels et agricoles sont pénalisantes, surtout pour les communes d'Andalousie où la production était concentrée. Les processus économiques associés à la baisse de production ne devraient pas être modifiés par l'amendement de la NRC de juin 2008. L'amendement concerne l'obligation de récolter mais sans l'exigence d'un rendement minimum. La résorption de la production cotonnière en Espagne devrait se poursuivre.

Mots clés : coton ; Espagne ; PAC ; politique agricole.

Thèmes : productions végétales ; économie et développement rural.

\section{Abstract \\ Effects of the Cotton Reform in Spain}

This paper analyzes the effects of the New Cotton Reform (NCR) in Spain for 2007-2008, two years after its application but before its amendment in June 2008. The particularity of this analysis is that it highlights the processes underlying the production decline. It is based on the exploitation of official statistics collected by the Andalusia Region and information obtained during the interviews of cotton sector stakeholders. These interviews were conducted so as to have cotton growers elaborate on the eventual implications of the NCR on their cultivation practices. This approach avoids having to build complex mathematical models to capture the impact of the NCR on production modes and outcomes. While cotton production was rather stable during the 1996-2005 period, the application of the NCR has contributed to a decrease in cotton acreage by more than $30 \%$. The production diminution was higher, around $60 \%$, one year immediately after the application of the reform because of the collapse of seedcotton yield associated with a strong reduction of input use. This evolution is positive from the environmental standpoint, but employment losses in the industrial and agricultural sectors are harmful, notably to the municipalities where cotton production is concentrated. The economic processes underlying the production decline should not be reversed following the amendment of the NCR in June 2008. The amendment makes it compulsory for growers to harvest but without any requirement of minimum yield. The production decline in Spain should continue.

Key words: agricultural policies; CAP; cotton; Spain.

Subjects: economy and rural development; vegetal productions. 
e coton est la principale culture arable irriguée dans le sud de l'Espagne, avec une moyenne d'environ 92000 hectares (période 1999-2003) cultivés dans 9200 exploitations. La culture du coton y revêt une importance sociale par les emplois qu'elle génère, estimés à 1,47 million de jourshommes (Consejería de Agricultura y Pesca, 2005). Comme dans tous les pays cotonniers, la production de coton est liée aux secteurs des services (fournisseurs d'intrants) et de l'industrie (égrenage). Le secteur coton impliquait 27 égreneurs avant la nouvelle réforme cotonnière (NRC) qui fut décidée par le règlement n 864/2004 du 29 avril 2004 du Conseil européen (CE) et mise en application en 2006. Un élément clé de la réforme concerne le découplage partiel de la subvention.

L'évaluation ex ante des impacts de la NRC a fait l'objet de peu de travaux. Une baisse de la production européenne était envisagée officiellement et elle servait de preuve de la bonne volonté de l'Union européenne dans les négociations à l'Organisation mondiale du commerce (OMC), notamment à l'endroit des interlocuteurs africains (Mandelson, 2005). Une baisse drastique de la production est anticipée, quoique le niveau de la baisse dépende de l'évolution du prix mondial (Araujo-Bonjean et al., 2006). Une étude a abordé directement l'incidence sur les producteurs dont le bien-être devrait être d'autant plus amélioré que le prix du coton augmente avec la baisse de la production (Karagiannis, 2004). Depuis la mise en œuvre de la NRC, des études ont indiqué que le découplage induisait une baisse de la production en Grèce (Katranidis et Kotakou, 2008) alors que le risque de disparition de la production cotonnière en Espagne était signalé (Arriaza, 2008; Arriaza et Gomez-Limon, 2006; Arriaza et al., 2006).

Cet article analyse les effets de la NRC en Espagne jusqu'à la campagne 2007-2008, soit deux ans après son application mais avant son amendement en juin 2008, à la suite d'ailleurs de la contestation du gouvernement espagnol. Il se distingue des travaux antérieurs par une meilleure mise en évidence des processus en ouvre dans la baisse constatée de la production.

L'étude est réalisée à partir des statistiques officielles tenues par la Province d'Andalousie et des informations recueillies au cours d'entretiens avec les acteurs du secteur coton ${ }^{1}$. Au cours des réformes successives de la Politique agricole com- mune (PAC), les pays européens ont dû mettre en place des systèmes d'information pour la supervision de l'aide octroyée par l'Union européenne. Dans le cas du coton, un tel système d'information est opérationnel et fiable ; il intègre les données des producteurs et des productions depuis la campagne 1999-2000 (Granado et al., 2009a). L'étude réalisée s'appuie par ailleurs sur les informations recueillies lors d'entretiens avec les acteurs du secteur coton, c'est-à-dire les producteurs et 26 non-producteurs (égreneurs, fournisseurs d'intrants et services d'appui technique). Ces entretiens avaient pour but de cerner comment les acteurs du secteur coton avaient compris la NRC et comment ils s'y sont adaptés; les discussions étaient poussées jusqu'à faire préciser par les producteurs les conséquences éventuelles de la NRC sur leurs pratiques culturales. Les informations recueillies permettent ainsi de se passer de la construction de modèles mathématiques très complexes pour détecter des causalités dans les corrélations observées à travers l'analyse des statistiques.

L'article est organisé en trois parties. La première partie retrace l'évolution de la réforme de la politique cotonnière dans l'Union européenne jusqu'à l'amendement de juin 2008. La deuxième partie est consacrée aux effets de la NRC sur la production et les pratiques culturales. La troisième partie souligne les impacts socio-économiques et industriels. La conclusion récapitule les principaux facteurs de baisse de la production cotonnière en Espagne et suggère que le récent amendement de la NRC aura peu d'effets pour enrayer le déclin observé de la production en Espagne.

\section{La NRC}

\section{et son amendement}

\section{Évolution de la politique cotonnière jusqu'à la NRC}

L'évolution de la politique cotonnière en Europe a été rappelée et replacée dans le contexte de la construction européenne

\footnotetext{
${ }^{1}$ Ces entretiens font partie des travaux conduits dans le cadre du Projet ISSCRI, financé par la Commission européenne et coordonné par le Centre de coopération internationale en recherche agronomique pour le développement (Cirad).
}

avec l'entrée de la Grèce dans la Communauté européenne en 1981 (Botonaki et al., 2009). Cette entrée marque aussi le début de la politique cotonnière en Europe ; le protocole $\mathrm{N}^{\mathrm{O}} 4$ annexé à l'Acte d'adhésion de la Grèce obligeant à soutenir la production de coton dans les régions où elle revêt une grande importance économique et sociale.

Jusqu'à la réforme de 2004, les producteurs de coton de l'Union européenne, c'est-à-dire en Grèce et en Espagne principalement, étaient soutenus par la garantie d'un prix d'achat du coton-graine bien supérieur au prix mondial. Dans la pratique, les égreneurs jouaient un rôle primordial dans le système de soutien car ils étaient chargés de payer le prix soutenu aux producteurs. Ce système était très incitatif et il était responsable d'une forte augmentation de la production. La croissance annuelle de la production dans les deux pays a été estimée à 7,3\% depuis la mise en ouvre de la politique de soutien jusqu'aux années 1990 (Baffes, 2005). Grâce à cette politique, la Grèce est devenue exportateur structurel (Fok, 1997). En Espagne, le record de production a été enregistré trois ans après l'entrée dans la Communauté européenne (Dorey, 1992). Le souci de contrôle budgétaire au niveau européen a conduit ensuite à définir des quotas de production et à appliquer des soutiens dégressifs en cas de dépassement des quotas. La politique cotonnière a été maintes fois ajustée pour modifier les modes de calcul des quotas et de la dégressivité du prix garanti en cas de dépassement. Avant la réforme plus fondamentale de 2004, la politique cotonnière avait déjà intégré des mesures d'incitation pour la constitution de coopératives de producteurs. L'incitation à adopter des pratiques culturales plus respectueuses de l'environnement était déjà globalement mise en place dans la réforme générale de la politique agricole commune (PAC) à la fin des années 1990. En 2004, la réforme décidée visait à aligner la politique cotonnière sur les principes généraux de la réforme de la PAC. Elle passa du soutien des prix au soutien du revenu, la période 2000-2002 étant prise comme période de référence pour calculer les aides à verser. Le nouveau régime du coton octroie l'aide en combinant paiement découplé et couplé. Le paiement découplé est un paiement direct à l'exploitation, non lié à la production ; il est fixé de manière à constituer $65 \%$ de la part nationale de l'aide destinée aux producteurs de 
coton. Le second paiement, ou aide couplée, est lié à la surface cultivée pour le coton et il représente les $35 \%$ restants de l'aide totale au coton. La seule condition pour bénéficier du paiement couplé est que les producteurs doivent mener la culture du coton jusqu'à l'ouverture des capsules. Pour ce qui concerne le paiement découplé, les producteurs doivent s'engager à opter pour des techniques culturales moins néfastes pour l'environnement et la santé publique.

En Espagne, le paiement découplé est fixé à 1509 euros/ha, pour une surface éligible de 70000 hectares alors que le paiement couplé peut atteindre 1039 euros/ha. La condition relative à l'ouverture des capsules est cependant assez peu contraignante pour percevoir le paiement couplé. Elle peut encourager une production extensive, voire un semi-abandon de la production de coton, avec une réduction considérable de l'emploi d'intrants (engrais, pesticides et eau d'irrigation) et la non-récolte du produit.

\section{La nouvelle version de la NRC}

Dès sa mise en œuvre en 2006, la NRC provoqua une contestation en Espagne qui aboutit à son amendement en juin 2008. En Grèce, il n'y eut pas de réaction car la coordination entre les acteurs du coton y est inexistante depuis le démantèlement du Cotton Board à la fin des années 1990 (Botonaki et al., 2009).

La contestation vint d'abord des acteurs du secteur coton de la Région d'Andalousie. Elle fut reprise par le gouvernement de cette Région, ce qui obligea le gouvernement espagnol à contester la NRC à son tour, alors qu'il y était favorable.

Prévoyant un effondrement de la production et donc une chute drastique de leurs activités, les égreneurs ont joué un rôle aussi important que les producteurs de coton pour contester la NRC dans les modalités décidées en 2004. Les producteurs agricoles en Espagne sont regroupés au sein de trois organisations principales en fonction notamment de la taille des exploitations : les "gros" producteurs dans l'ASAJA (Asociación Agraria de Jóvenes Agricultores), les producteurs de polyculture dans le COAG (Coordinadora de Organizaciones de Agricultores y Ganaderos) et les " petits " producteurs dans l'UPA (Unión de Pequeños Agricultores y Ganaderos). Ces organisations différaient cependant quant au type d'amendement souhaité. L'ASAJA et l'UPA souhaitaient un retour au régime d'avant la réforme ou la formule du découplage total. Le COAG préférait en revanche un découplage limité à $20 \%$. La coordination de la contestation a abouti à la constitution d'une plate-forme multiacteurs appelée Mesa del algodón, incluant le gouvernement de la Région d'Andalousie, et a réussi à faire pression sur le gouvernement espagnol pour qu'il introduise une action en justice.

La plainte du Royaume d'Espagne contre le Conseil européen pour l'adoption de la NRC a été jugée en septembre 2006 par la Cour de justice européenne. Le jugement, sans remettre en cause les principes de la réforme engagée, conteste l'équité de la décision prise en l'absence d'étude suffisante sur les impacts de la réforme retenue. L'évaluation de la rentabilité pour les producteurs a été jugée imparfaite en raison de la prise en compte inadéquate de la maind'œuvre familiale. La Commission européenne est surtout jugée fautive pour l'absence de considération des impacts de la NRC sur le secteur de l'égrenage sans lequel la production cotonnière ne peut se poursuivre. Le jugement suspend la NRC jusqu'à ce que la Commission Européenne soumette une nouvelle proposition après réalisation d'une étude d'impact complémentaire.

L'étude a été menée au cours du premier semestre de 2007, un an après l'application de la NRC. Les résultats confirment les éléments fournis dans la plainte de l'Espagne (LMC International, 2007) quant aux impacts négatifs dans le secteur de l'égrenage. L'étude réalisée a également comparé trois options d'amendement de la NRC. Sur la base de cette étude, la Commission européenne a soumis en novembre 2007 une nouvelle version de la NRC qui fut adoptée en juin 2008. La seule modification concerne l'obligation de récolter le coton pour toucher le paiement couplé, avec le respect d'une qualité minimale du coton à livrer.

\section{Impacts}

\section{sur la production et les pratiques culturales}

\section{Évolution de la surface et de la production cotonnières}

L'évolution de la production cotonnière en Espagne est irrégulière depuis son entrée dans l'Union européenne. La période de sécheresse très sévère entre 1993 et 1995 a fait plonger la production une première fois. Cette production a remonté par la suite pour se stabiliser autour de 300000 tonnes de coton-graine à partir d'une superficie moyenne de 91000 hectares entre 1996 et 2005. L'application de la NRC marque un nouvel épisode de forte baisse de la production, à partir d'une superficie tombéeà environ 60000 hectares (figure 1), au cours d'une période à pluviométrie plus faible que la normale.

Avant la NRC, l'Andalousie représentait $97 \%$ de la production espagnole totale. Depuis la NRC, cette part a atteint $100 \%$ car la production a totalement disparu ailleurs dans le pays.

Durant toute la période de couplage des subventions (1986-2005), production et superficie cotonnière ont progressé parallèlement, à un rythme similaire (figure 1). L'Espagne faisait partie du petit groupe de pays producteurs obtenant un rendement de coton-graine supérieur à $3000 \mathrm{~kg} / \mathrm{ha}$. Depuis la mise en ouvre du système découplé, la courbe de la production a chuté rapidement en raison de l'effondrement des rendements moyens, à un tiers du niveau atteint avant la NRC (figure 2). Les rendements du coton qui variaient entre 3100 et $4000 \mathrm{~kg} / \mathrm{ha}$ de cotongraine entre 2000 et 2005, sont passés à 2 321, 1862 et $1048 \mathrm{~kg} / \mathrm{ha}$ de cotongraine de 2006 à 2008. La chute de rendement est bien plus prononcée que celle de la consommation de l'eau d'irrigation (figure 2), suggérant qu'elle est alimentée par un changement de pratiques culturales des producteurs ( $c f$. infra).

\section{Abandon pour les cultures concurrentes en Andalousie}

Avant la NRC, le coton était la culture arable la plus importante dans l'agriculture irriguée en Andalousie, les autres cultures étant le maïs, le blé, le tournesol et le riz (figure 3). Les surfaces consacrées au coton, culture annuelle, étaient cependant dépassées par deux cultures pérennes (agrumes et olivier).

Après l'application de la NRC, la superficie en coton a baissé de 25000 hectares qui ont été consacrés en grande partie au blé et au tournesol. Il convient de noter que la baisse de superficie est également enregistrée pour la betterave (qui a également subi une réforme radicale du régime de soutien) ainsi que pour le maïs et le riz (cultures plus sensibles à la sécheresse). 


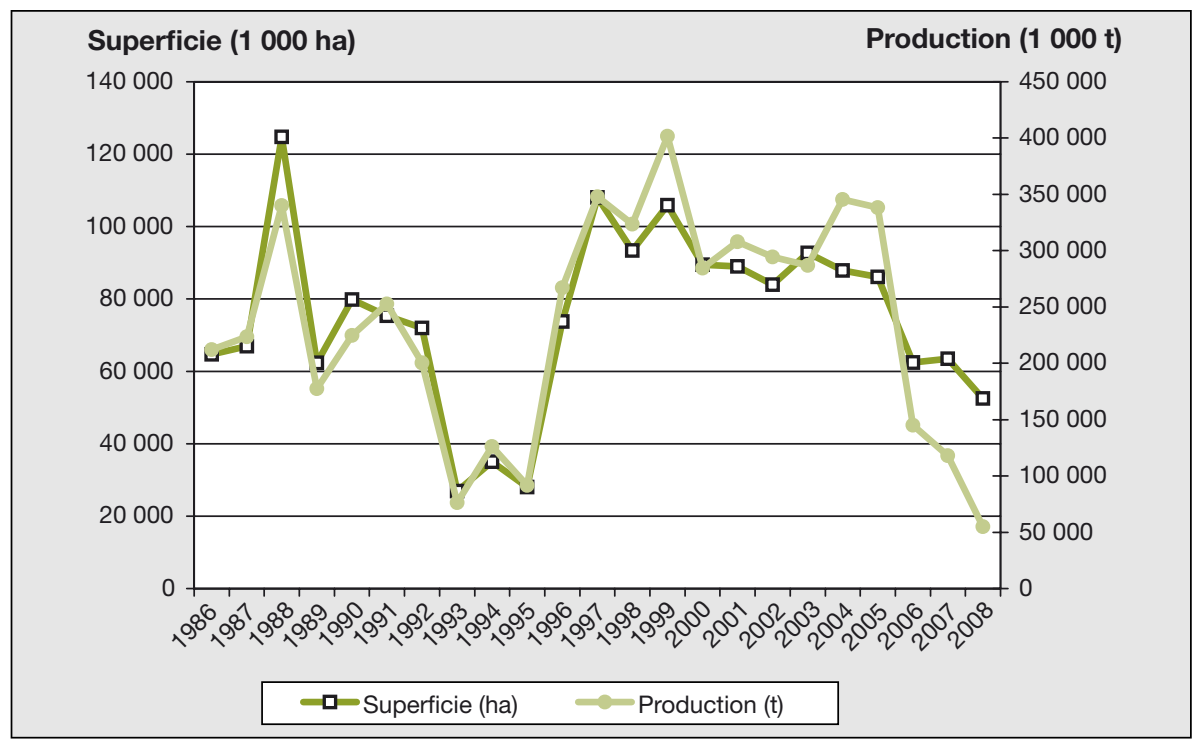

Figure 1. Évolution de la superficie et de la production de coton en Espagne.

Figure 1. Evolution of cotton area and production in Spain. Source : Ministerio de Medio Ambiente y Medio Rural y Marino.

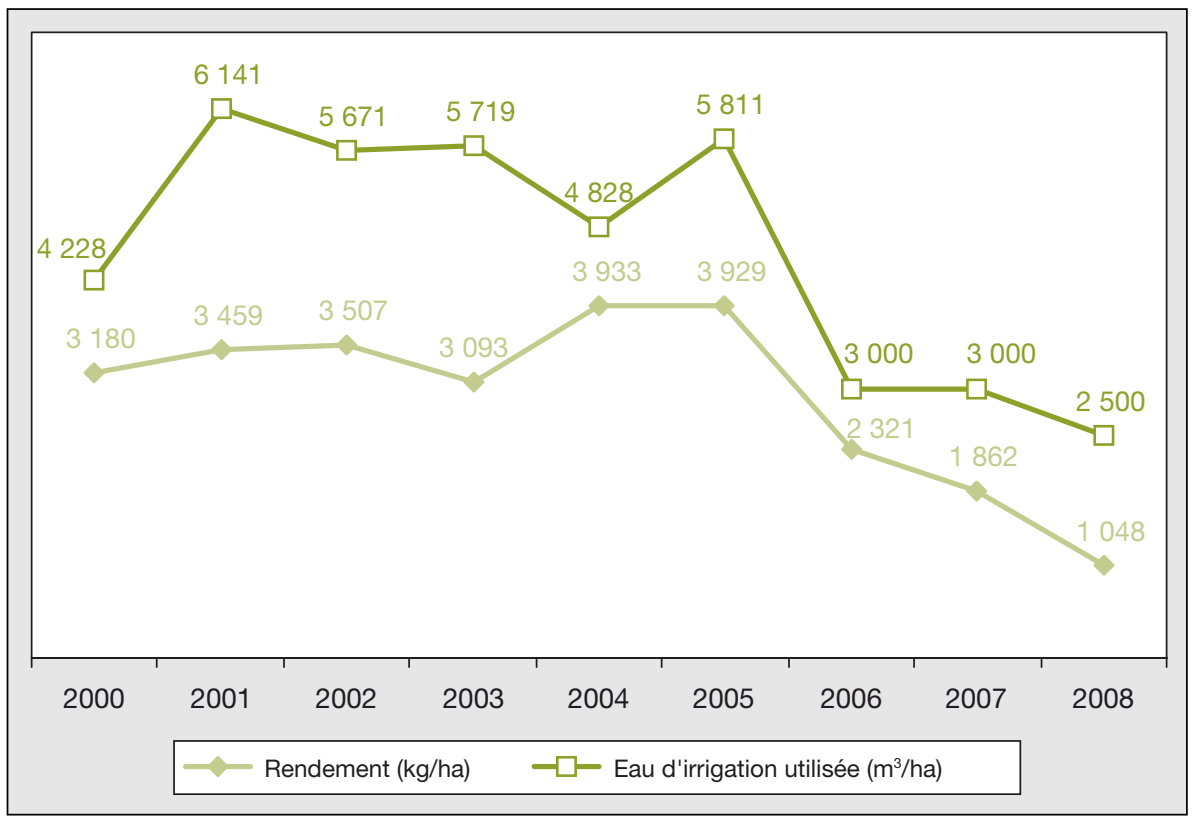

Figure 2. Évolution des rendements du coton durant la période 2000-2008.

Figure 2. Evolution of seedcotton yield during the 2000-2008 period.

\section{Incidence de la NRC sur la baisse de production}

Un modèle simple de régression permet de confirmer l'incidence de la NRC sur la baisse de la production de coton. Le modèle considère que le niveau de production de coton dépend linéairement de la superficie cultivée (variable Sup.), de la disponibilité en eau (variable Eau) et du type de mécanisme de soutien (variable Découplé).

La faible disparité de structure entre les exploitations et la faible taille de ces dernières, avec une sole moyenne de coton de moins de 10 hectares (Granado et al., 2009a) rendent réaliste l'hypothèse d'une absence de rendement d'échelle et justi- fient le choix d'un modèle linéaire. La variable "Découplé " correspond non seulement au changement de régime de soutien, mais elle intègre aussi les modifications de pratiques culturales pouvant découler du changement de régime.

L'application des données officielles de la Province d'Andalousie au modèle aboutit à l'équation (1) suivante, dont les coefficients sont très significatifs comme l'indique le tableau 1 :

Production $=90894+1,046 *$ Sup.

$+21,37 *$ Eau-107 590*Découplé

Selon l'équation (1), l'impact de la baisse de la superficie sur la production est la plus faible. La réduction de la disponibilité en eau, passant de $5500 \mathrm{~m}^{3} / \mathrm{ha}$ niveau considéré comme correspondant à une année normale - à environ $2500 \mathrm{~m}^{3} /$ ha après l'application de la $\mathrm{NRC}$ - aurait induit une chute de la production de 64110 tonnes, bien plus faible que celle qui résulte du mécanisme découplé (107590 tonnes).

\section{Évolution des pratiques agricoles d'intensification réduite}

Dès avant la mise en ouvre de la NRC, un début de réduction du recours aux intrants agricoles était observé (figure 4). La notion de "production intégrée " était promue pour inciter les producteurs à utiliser moins d'intrants chimiques. Avec la réforme de la PAC, l'Espagne a mis en place le paiement d'une incitation, appelée mesure agri-environnementale, allouée aux producteurs qui s'engageaient dans la "production intégrée". Dès 2007, les trois quarts des producteurs ont adhéré au nouveau mode de production. Parmi les règles à respecter, les producteurs devaient cesser la technique de semis sous plastique qui permettait des semis plus précoces (et donc aussi une espérance de rendement plus élevée). Le recours aux engrais était fortement abaissé (tableau 2), surtout pour ce qui concerne l'azote dont la dose maximale admise devait être inférieure à 120 unités fertilisantes par hectare, soit moitié moindre que ce qui était pratiqué en "culture conventionnelle". Le tableau 2 montre qu'en réalité les producteurs ont utilisé des quantités bien moindres d'engrais après l'application de la NRC, attestant d'une démarche délibérée d'extensification et de réduction des coûts. Le recours 
Tableau 1. Régression linéaire sur les facteurs de la baisse de la production.

Table 1. Linear regression of production decline factors.

\begin{tabular}{lcccc}
\hline Variables & Coefficients & Écart type & t Student & $\begin{array}{c}\text { Probabilité } \\
\text { de non- } \\
\text { signification }\end{array}$ \\
\hline Constante & 90894,4 & 29178,5 & 3,12 & 0,0057 \\
SUP & 1,04591 & 0,39270 & 2,66 & 0,0154 \\
EAU & 21,3666 & 4,81285 & 4,44 & 0,0003 \\
Découplé & -107590 & 32274,6 & $-3,33$ & 0,0035 \\
\hline
\end{tabular}

Source des données : 1986-2008.

Superficie en Andalousie (1 000 ha)

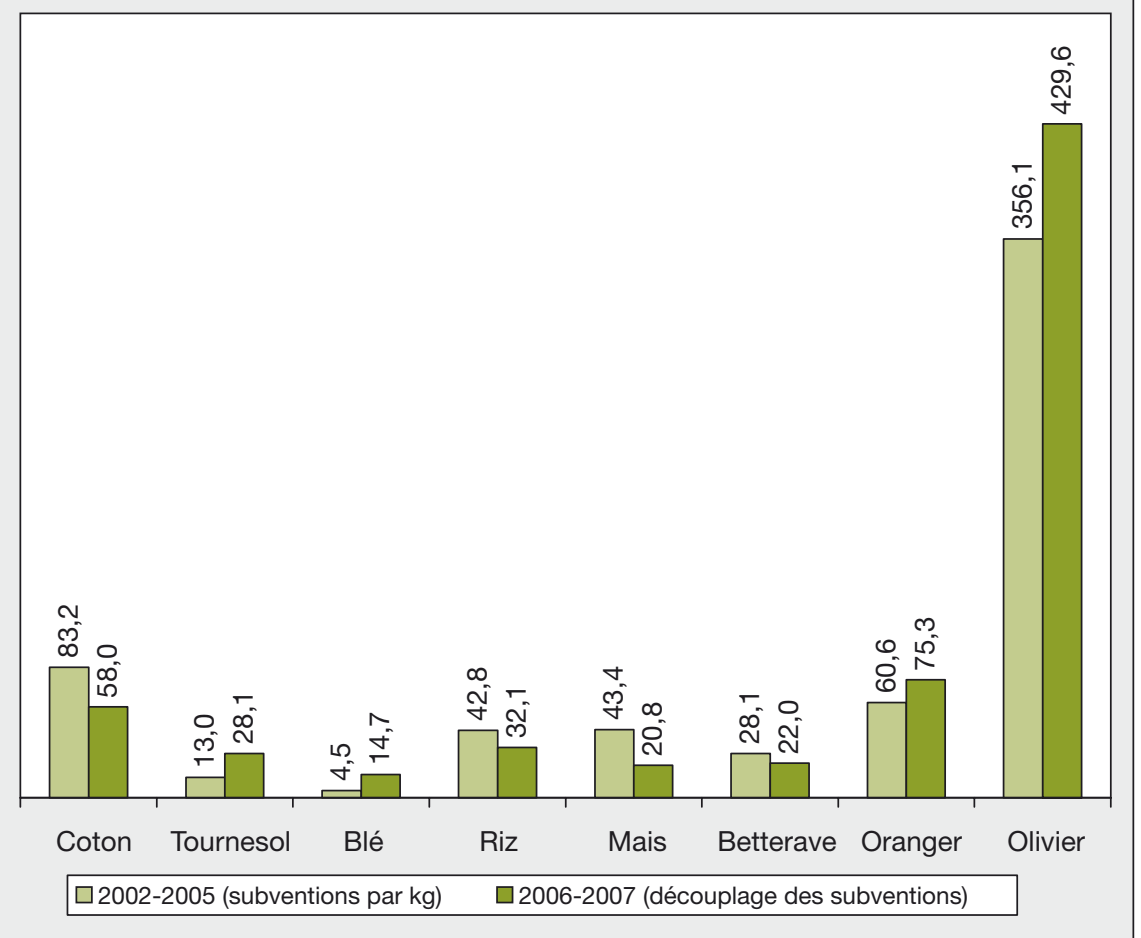

Figure 3. Superficie des principales cultures irriguées en Andalousie.

Figure 3. Acreages of the main irrigated crops in Andalusia. Source : Consejería de Agricultura y Pesca. Junta de Andalucía.

aux pesticides, notamment aux herbicides, devait être aussi réduit. Cette option explique la fréquence de champs de coton totalement enherbés lors des deux campagnes 2006-2007 et 2007-2008.

Les pratiques culturales d'extensification évoquées ci-dessus expliquent la forte baisse des coûts directs totaux par hectare de coton, - $42 \%$, comme on peut le voir dans la figure 4. Elles permettent aussi de comprendre l'effondrement observé du rendement qui découle d'une espérance de rendement réduite par des semis plus tardifs et des apports d'engrais moindres ainsi que d'une protection insuffisante contre les adventices ou les ravageurs.

La tendance d'extensification parait bien ancrée. Selon les producteurs rencontrés en 2007 , la poursuite de la culture cotonnière va se faire avec une baisse de l'utilisation des intrants chimiques (tableau 3).
Impacts

\section{socio-économiques et industriels}

Ces impacts concernent l'environnement, la résorption de l'industrie d'égrenage du coton-graine et l'incidence sur l'économie locale de l'Andalousie.

\section{Impacts environnementaux}

Sur le plan environnemental, l'impact de la NRC est positif du fait de la diminution importante de l'utilisation des intrants chimiques. La pollution par le plastique utilisé pour le semis est arrêtée. La pollution des nappes phréatiques par l'azote des engrais est fortement réduite.

Un autre effet positif, mais plus subtil, concerne une meilleure efficience de l'utilisation de l'eau d'irrigation. C'est le résultat combiné d'une réallocation des terres irriguées entre les cultures, d'une part, et, d'autre part, d'une réduction de l'intensité de l'irrigation des cultures de coton (Granado et al., 2009b; Lorite et Arriaza, 2009). Ce changement dans l'irrigation participe aussi à la démarche d'extensification qui se détourne de la maximisation des rendements recherchée auparavant.

\section{Impacts sur l'industrie d'égrenage}

Ces impacts sont bien rapportés dans l'étude d'impact commanditée par la Commission européenne en 2007, étude pour laquelle les autorités provinciales de l'Andalousie, y compris l'auteur de cet article, ont fourni les données nécessaires.

Dès 2007, il était observé qu'en raison de la baisse de la production de $60 \%$ les usines d'égrenage n'ont fonctionné, en moyenne, qu'à $40 \%$ de leurs capacités, augmentant fortement le coût fixe unitaire de transformation. Le coton livré est davantage chargé en impureté, ce qui a diminué d'un point le rendement à l'égrenage. En conséquence, le coût moyen d'égrenage a augmenté de $60 \%$, en passant de 92,69 à 148 euros/t. Alors que toutes les usines d'égrenage opéraient au-dessus du seuil de rentabilité avant la NRC, une seule d'entre elles a franchi ce seuil en 2006-2007. Au cours de cette campagne, cinq usines sont restées inactives tandis qu'une entreprise a définitivement fermé. La baisse globale d'activité des usines d'égrenage a induit une perte de 1900 journées de travail permanent et de 376 postes temporaires. Le préjudice économique subi par le sec- 


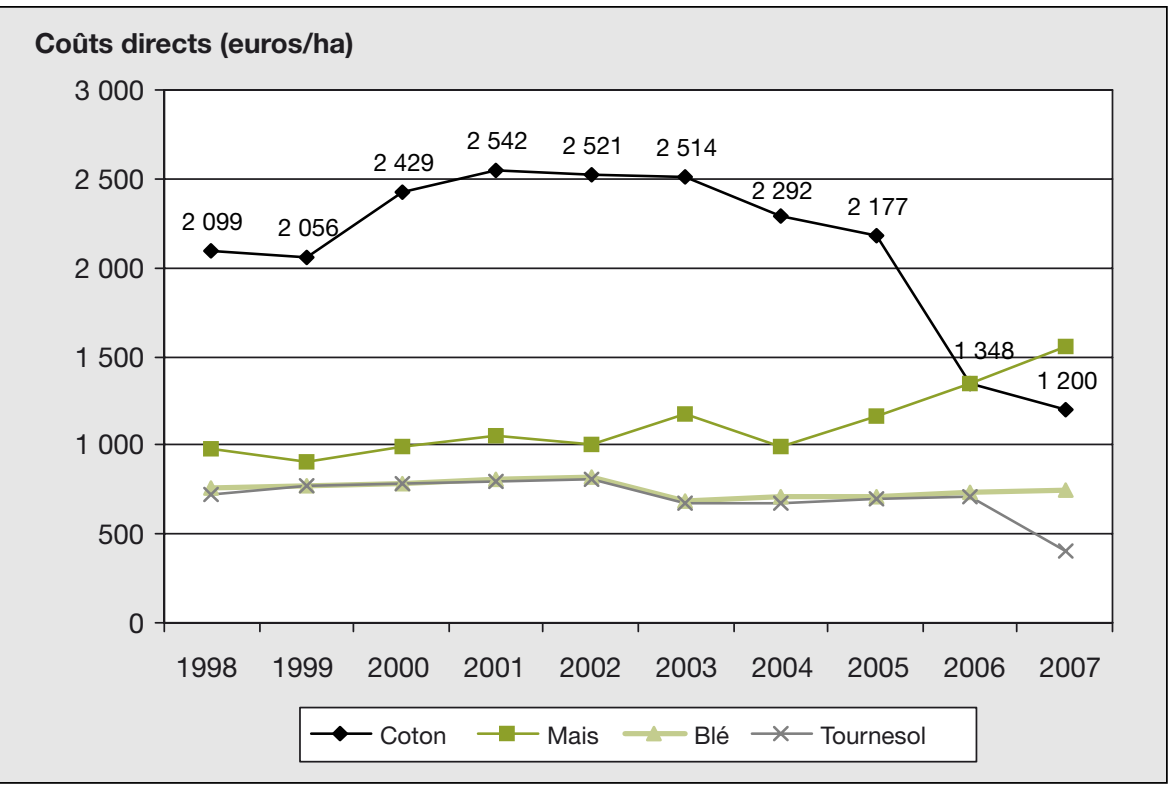

Figure 4. Évolution des coûts directs de la culture du coton.

Figure 4. Evolution of the direct costs of cotton cropping.

Source : Arriaza, 2008.

\section{Tableau 2. Comparaison des intrants nécessaires par mode de production.}

Table 2. Comparison of needed inputs according to production systems.

\begin{tabular}{lccc}
\hline & $\begin{array}{c}\text { Dose observée } \\
\text { en production } \\
\text { conventionnelle } \\
\text { (avant la NRC) }\end{array}$ & $\begin{array}{c}\text { Dose maximale } \\
\text { autorisée } \\
\text { en production intégrée } \\
\text { (après la NRC) }\end{array}$ & $\begin{array}{c}\text { Doses } \\
\text { observées } \\
\text { en 2006 }\end{array}$ \\
\hline Azote & $200-250 \mathrm{UF} / \mathrm{ha}$ & $118,3 \mathrm{UF} / \mathrm{ha}$ & $90 \mathrm{UF} / \mathrm{ha}$ \\
Phosphore & $120 \mathrm{UF} / \mathrm{ha}$ & $96 \mathrm{UF} / \mathrm{ha}$ & $30 \mathrm{UF} / \mathrm{ha}$ \\
Potasse & $75-125 \mathrm{UF} / \mathrm{ha}$ & $96 \mathrm{UF} / \mathrm{ha}$ & $25 \mathrm{UF} / \mathrm{ha}$ \\
\hline
\end{tabular}

Source: informations extraites d'entretiens avec des techniciens cotonniers en 2007. UF : unité fertilisante.

teur de l'égrenage est estimé à plus de 25 millions d'euros.

La perte de rentabilité de l'industrie de l'égrenage procède en réalité de plusieurs processus socio-économiques que l'étude commanditée par la Commission européenne a omis de préciser.

La réforme du régime du coton a exercé un effet immédiat non seulement sur la quantité de coton-graine que les égreneurs ont reçu, mais aussi sur la qualité de celui-ci. Concernant la qualité, le nouveau système n'encourage pas suffisamment les producteurs à livrer du coton dans les meilleures conditions de pro- preté $^{2}$ et d'humidité. La prise en compte de la qualité ne peut s'appliquer qu'au paiement couplé $(35 \%)$ alors que la majeure partie de l'aide, le paiement découplé (65\%), est indépendante de la qualité du coton livré aux égreneurs. Du point de vue industriel, un degré d'humidité supérieur se traduit par des coûts énergétiques plus élevés lors du processus de séchage. Par ailleurs, une charge

\footnotetext{
${ }^{2}$ La différence avec les prix mondiaux pour chaque catégorie n'est pas suffisamment élevée pour encourager une production de coton de qualité maximale dans le système productif espagnol.
}

forte en impuretés du coton-graine conduit à un coton de moindre qualité après égrenage, et ce au prix d'un coût accru de nettoyage et d'usure des scies des égreneuses.

Avec la NRC, les égreneurs ont perdu des garanties dans le recouvrement de certains coûts d'opération. Le paiement de 5,31 euros pour 100 kilos de cotongraine (la différence entre le prix objectif et le prix minimum payé à l'agriculteur) qui était versé aux égreneurs est supprimé ; il était destiné à couvrir les frais de transport du coton-graine de l'exploitation à l'usine ainsi que les frais d'égrenage. Les égreneurs sont par ailleurs soumis directement aux fluctuations du prix du coton-fibre ; le décalage dans le temps entre la vente du coton-fibre et l'achat du coton-graine peut induire un déficit si le prix mondial a baissé après l'achat aux producteurs.

Depuis l'application de la NRC, le coût du transport du coton-graine est supporté par les producteurs. L'importance de ce coût - qui est fonction de l'éloignement du producteur d'une usine d'égrenage, mais aussi de la capacité du producteur à organiser le transport - a une incidence sur la décision du producteur à récolter et à livrer ou non son coton. Ce phénomène explique que l'effet de la baisse de la production cotonnière est inégalement réparti entre les égreneurs. Les égreneurs situés dans les zones à plus grande concentration de la production ont été moins pénalisés. Cela explique pourquoi certaines usines d'égrenage ont cessé temporairement ou définitivement leurs activités dès 2006.

\section{Impacts négatifs sur l'économie locale}

La majeure partie du coton était cultivée dans quelques communes du sud de l'Espagne, comme le montre la figure 5 . Audelà de la répercussion économique globale du recul du coton, l'impact socioéconomique à court terme dans ces communes est encore plus marqué par la perte d'emplois saisonniers agricoles et industriels.

Pour ces communes du sud de l'Andalousie, contrairement au centre et au nordest, il existe peu de cultures alternatives en raison de la salinité des sols. Cette région avait déjà subi un choc économique avec la réforme européenne dans le secteur de la betterave sucrière. La NRC provoque un choc encore plus important 
Tableau 3. Perception des producteurs sur l'évolution du recours aux facteurs de production à l'avenir (\% réponses).

Table 3. Growers' opinions on the use of production factors in the future ( $\%$ of answers).

\begin{tabular}{lcccccc}
\hline & $\begin{array}{c}\text { Forte } \\
\text { hausse }\end{array}$ & $\begin{array}{c}\text { Faible } \\
\text { hausse }\end{array}$ & Stable & $\begin{array}{c}\text { Faible } \\
\text { baisse }\end{array}$ & $\begin{array}{c}\text { Forte } \\
\text { baisse }\end{array}$ & Total \\
\hline Investissement en équipements & 7,7 & 7,7 & 73,1 & 3,8 & 7,7 & 100,0 \\
Emploi d'engrais & 0,0 & 0,0 & 34,6 & 26,9 & 38,5 & 100,0 \\
Emploi de pesticides & 0,0 & 0,0 & 34,6 & 23,1 & 42,3 & 100,0 \\
Emploi de semences de qualité & 0,0 & 3,8 & 65,5 & 19,2 & 11,5 & 100,0 \\
\hline
\end{tabular}

Source : informations extraites de 26 entretiens avec des exploitants cotonniers en 2007.

car le coton tenait une place économique plus grande que la betterave sucrière.

Avant la NRC, la production de coton représentait $5,5 \%$ de la valeur totale de la production agricole dans trois provinces (Cordoue, Cadix et Jaén) et $9 \%$ dans le cas de Séville. La mise en ouvre de la NRC a entraîné une réduction de la part du coton dans le secteur agricole, comme illustré sur la figure 6.

En termes de contribution à la valeur de la production agricole, le coton et la betterave sucrière ont baissé, les mêmes causes de réforme du régime de soutien induisant les mêmes effets. Cette baisse de la contribution relative est accentuée par l'augmentation des prix des céréales et des oléagineux qui ont accru les valeurs de ces derniers produits. Maintenant que cette augmentation de prix de ces produits est résorbée après une période de baisse, la baisse de la valeur totale de la production agricole en Andalousie va devenir plus visible.

\section{Impacts sociaux}

L'application de la NRC a eu un effet négatif sur l'emploi. L'effet sur l'emploi industriel est déjà évoqué dans l'analyse des effets sur l'industrie de l'égrenage. Les effets sur les emplois agricoles sont directement liés aux changements des pratiques culturales des producteurs de coton.

La production de coton est une culture assez exigeante en main-d'œuvre.

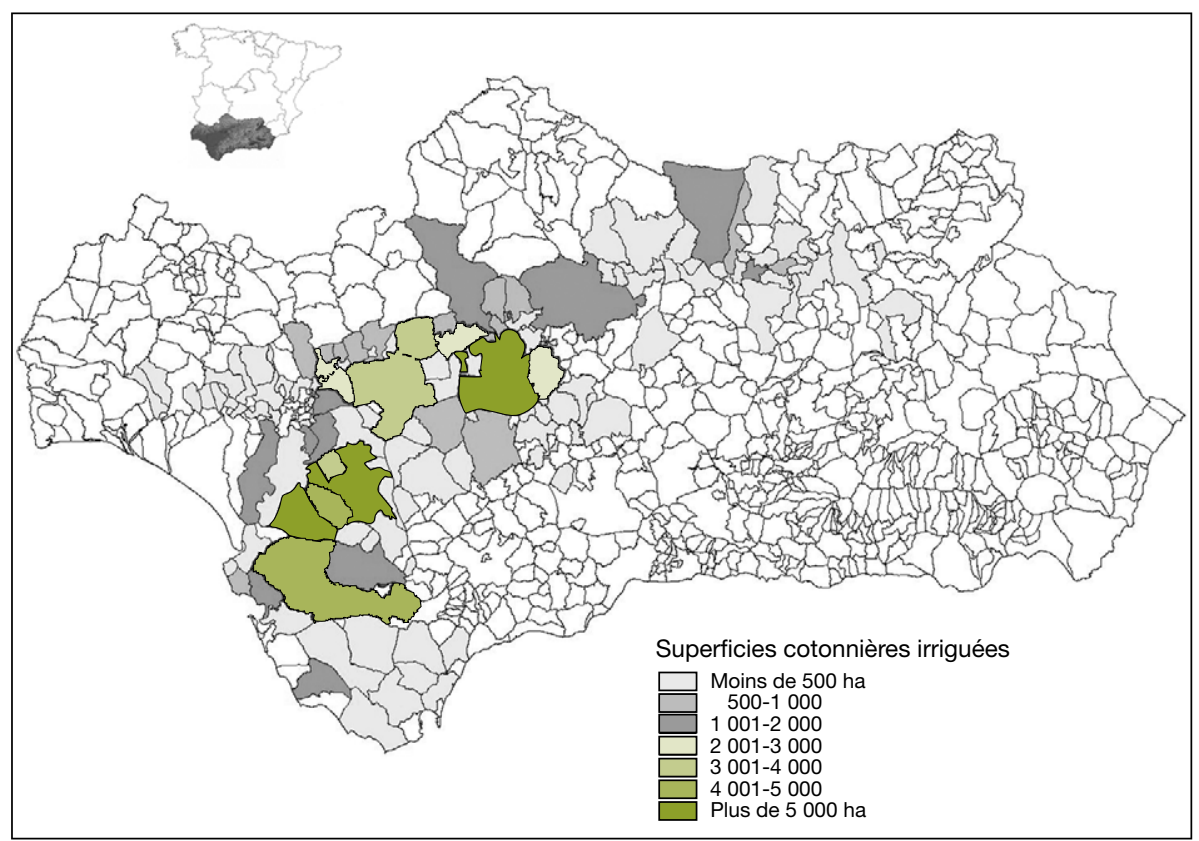

Figure 5. Localisation de la production cotonnière en Andalousie (2006).

Figure 5. Geographic distribution of cotton production in Andalusia. Source : Consejería de Agricultura y Pesca. Junta de Andalucía.
Comme pour d'autres facteurs de production, le recours à son utilisation a été réduit. Avant la NRC, la production de coton absorbait en Andalousie les deux tiers de la main-d'œuvre agricole totale employée dans les cultures arables irriguées (Consejería de Agricultura y Pesca, 2005). Cela équivaut à environ 5500 travailleurs à temps plein. Dans certaines communes du sud de l'Andalousie, elle emploierait jusqu'à $80 \%$ de la maind'œuvre agricole totale.

Après la NRC, le nombre de jourshommes a été réduit de $43 \%$, comme indiqué dans le tableau 4. Cette réduction résulte directement de plusieurs changements dans les pratiques culturales. L'arrêt de la technique de semis sous film plastique a réduit la demande en maind'œuvre; il en est de même quand on épand moins d'engrais et de pesticides. Le changement des programmes d'irrigation, en partie lié à la sécheresse, a conduit à réduire les tours d'arrosage qui sont consommateurs de maind'œuvre (pour le déplacement des tuyaux). Enfin, la décision de certains exploitants de ne pas récolter, surtout quand le prix du coton-graine n'est pas attrayant (cas de 2006) a une incidence directe sur le moindre besoin en maind'œuvre.

\section{Conclusion}

Depuis l'épisode de la sécheresse de 1993-1995, la production cotonnière en Espagne s'était stabilisée en fluctuant entre 300000 et 350000 tonnes de coton-graine au cours de la période 1996-2005. Avec l'application de la NRC en 2006, la superficie consacrée au coton a baissé de plus de $30 \%$, mais la baisse de la production a été encore plus forte, car le rendement moyen est 


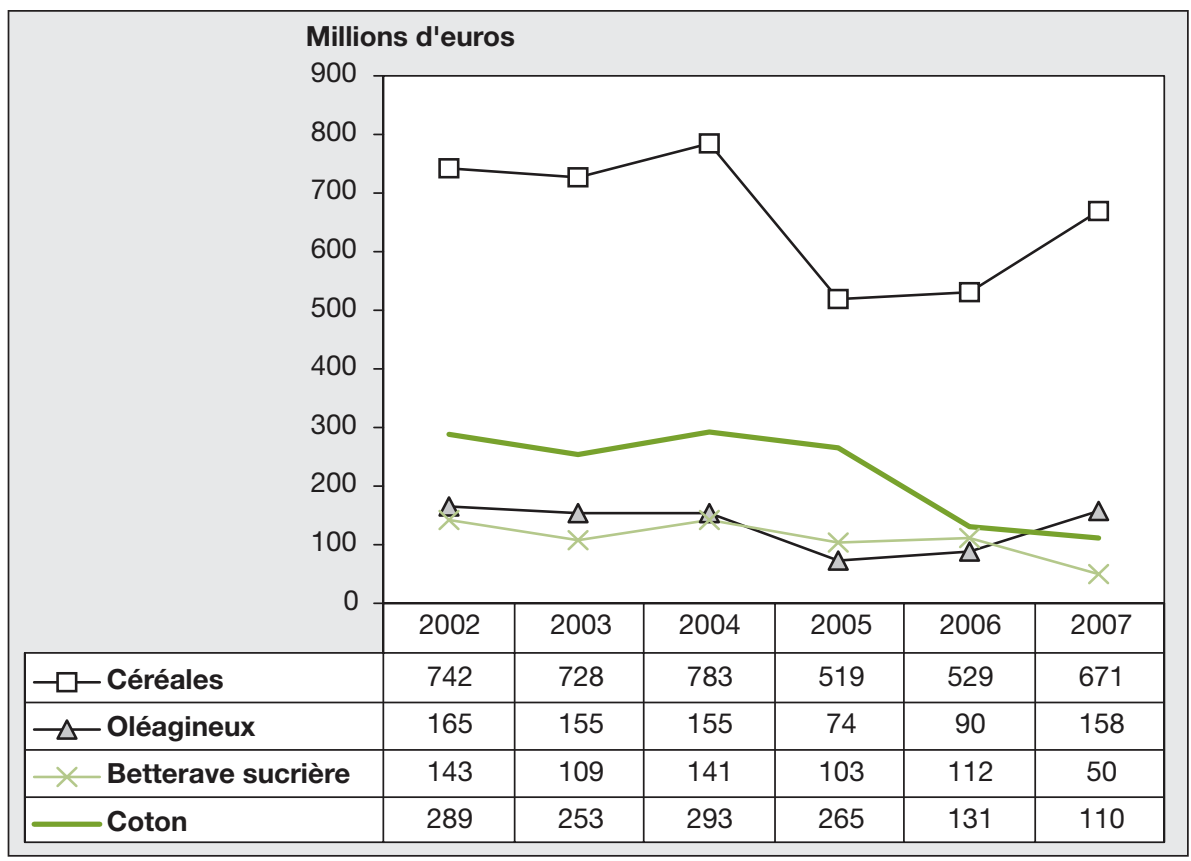

Figure 6. Évolution de la valeur de la production agricole en Andalousie.

Figure 6. Evolution of the agricultural production value in Andalusia.

Source : Consejería de Agricultura y Pesca. Junta de Andalucía.

tombé au tiers de la valeur antérieure. L'Espagne a aujourd'hui le niveau de rendement de l'Afrique de l'Ouest alors qu'elle figurait parmi les pays aux rendements les plus élevés.

La baisse du rendement est liée à un changement des pratiques culturales, orienté vers une réduction du recours aux intrants agricoles ou vers l'extensification. Les entretiens avec les producteurs indiquent que ce changement est associé à la NRC. Le modèle de régression linéaire confirme aussi l'incidence de la modification du régime de soutien; incidence qui est plus forte que la sécheresse qui s'est abattue sur l'Andalousie.

L'application de la NRC a eu un impact positif sur le plan environnemental, mais les autres effets sont plus pénalisants pour l'Espagne. La réduction de $60 \%$ de la production a fortement diminué la rentabilité des sociétés d'égrenage. La fermeture des usines d'égrenage est engagée dès 2006 et va se poursuivre, entraînant avec elle des pertes d'emplois permanents et saisonniers dans le secteur industriel. Cette baisse de rentabilité ne procède pas seulement de la réduction de la quantité de coton-graine à égrener, elle résulte aussi de plusieurs mesures de la NRC comme la suppression de la garantie de la couverture des coûts de transport du cotongraine et des frais d'égrenage, la perte d'une certaine protection contre les fluctuations du marché mondial du cotonfibre et le transfert du coût de transport du coton-graine aux producteurs.

La perte d'emplois agricoles est aussi conséquente. La réduction de ces emplois dans les exploitations de coton est estimée à $43 \%$ alors que le coton représentait la principale opportunité de travail pour les salariés agricoles.

Les effets économiques et sociaux de la NRC, aux plans agricole et industriel, sont importants. Ils le sont surtout pour les communes d'Andalousie où la production cotonnière est concentrée.

L'amendement de la NRC en juin 2008 n'a concerné que l'obligation de récolter le coton-graine pour toucher le paiement couplé. Ce changement ne modifiera pas les processus techniques et économiques engagés qui sont responsables de la baisse de la production. La plupart des producteurs livraient déjà leur coton même quand ils n'étaient pas obligés de récolter. Cet amendement n'ayant pas retenu la condition d'un rendement minimum en plus de l'obligation de récolte, l'on peut craindre que le déclin de la production cotonnière ne se poursuive en Espagne, avec ses effets socio-économiques négatifs, au moins à court terme.

\section{Tableau 4. Évolution du recours à la main-d'œuvre agricole par système d'irrigation et mode de culture.}

Table 4. Evolution of the use of agricultural labour according to irrigation and mulching modes.

\begin{tabular}{|c|c|c|c|c|c|c|c|}
\hline \multirow{2}{*}{$\begin{array}{c}\text { Système } \\
\text { d'irrigation }\end{array}$} & \multirow{2}{*}{$\begin{array}{l}\text { Mode } \\
\text { de culture }\end{array}$} & \multicolumn{3}{|c|}{$2003-2004-2005-2006$} & \multicolumn{3}{|c|}{ 2006-2007 } \\
\hline & & $\begin{array}{l}\text { Sup. coton } \\
\text { (ha) }\end{array}$ & $\begin{array}{c}\text { Jour- } \\
\text { homme/ha }\end{array}$ & $\begin{array}{l}\text { Total jours- } \\
\text { hommes }\end{array}$ & $\begin{array}{l}\text { Sup. coton } \\
\text { (ha) }\end{array}$ & $\begin{array}{c}\text { Jour- } \\
\text { homme/ha }\end{array}$ & $\begin{array}{l}\text { Total jours- } \\
\text { hommes }\end{array}$ \\
\hline Pluvial & Air libre & 2812 & 8,25 & 23205 & 3436 & 8,25 & 28,345 \\
\hline \multirow[t]{2}{*}{ Aspersion } & Plastique & 4026 & 20,65 & 83140 & - & - & - \\
\hline & Air libre & 10187 & 18,65 & 189989 & 14087 & 15,05 & 212,016 \\
\hline \multirow{2}{*}{$\begin{array}{l}\text { Goutte- } \\
\text { à-goutte }\end{array}$} & Plastique & 10117 & 18,65 & 188688 & - & - & - \\
\hline & Air libre & 16535 & 16,65 & 275311 & 12474 & 15,35 & 191,477 \\
\hline \multirow[t]{2}{*}{ A la raie } & Plastique & 22239 & 18,70 & 415872 & - & - & - \\
\hline & Air libre & 21988 & 16,70 & 367206 & 32819 & 13,70 & 449,624 \\
\hline Total & & 87906 & - & 1543411 & 62816 & - & 881,462 \\
\hline
\end{tabular}

Source : Consejería de Agricultura y Pesca. Junta de Andalucía. 


\section{Références}

Araujo-Bonjean C, Caplipel S, Traoré F Impacts des aides américaines et européennes sur le marché international de coton. Clermont-Ferrand: CERDI-Université d'Auvergne, 2006.

Arriaza M. Evaluación del impacto de la reforma del algodón en España. Cordoba (España): Consejería de Agricultura y Pesca, Junta de Andalucía, 2008.

Arriaza M, Gomez-Limon JA. How decoupling could mean dismantling of the cotton sector in Spain. New Medit Mediterranean Journal of Economics. Agriculture and Environment 2006 ; V : 4-14.

Arriaza M, Gomez-Limon JA, Gonzalez Arenas $J$, Ruiz Avilès P. Viability of the raw cotton pro duction in Spain after the decoupling of the subsidies. Proceedings 'XXVI Congess of the International Association of Agricultura Economists (IAAE)', Brisbane (Australia), 2006.

Baffes J. The "Cotton Problem". The World Bank Research Observer 2005 ; 20 : 109-44.
Botonaki A, Mattas K, Rozakis S, Tsiboukas K. Impact des évolutions de la politique européenne sur les décisions des producteurs de coton en Grèce. Cah Agric 2009; 18: 402-7. doi : 10.1684/agr.2009.0331

Consejería de Agricultura y Pesca. Diagnóstico del sector algodonero andaluz. Sevilla: Junta de Andalucía, 2005.

Dorey G. Espagne : un millénaire de coton. Coton et Développement 1992 ; (2) : 19-22.

Fok ACM. Etat, production et exportation cotonnières, industrie textile et développement économique. Une histoire économique du coton/Textile dans le monde. Doctorat en Economie, faculté des sciences économiques, université de Montpellier I, Montpellier, France, 1997.

Granado R, Mora JJ, Mendez MA, Recio EJ, Polonio D. Design of an information system for the characterisation of the Andalusian cotton farm. Life Sciences International Journal 2009a ; (NS) : 23-37.
Granado R, San Miguel P, Garcia R, Polonio D. Impact of the reforms of the cotton support scheme on Andalusia. Life Sciences International Journal 2009b ; (NS) : 38-49.

Karagiannis G. The EU cotton policy regime and the implications of the proposed changes for producer welfare. Rome : FAO, 2004.

Katranidis SD, Kotakou CA. Are CAP decoupling policies really production neutral? Proceedings 'International Congress of the European Association of Agricultural Economists', Ghent (Belgium), 2008.

Lorite IJ, Arriaza M. Effects of the EU agricultural subsidies on the irrigation management in Spain. Life Sciences International Journal 2009 ; (NS) : 140-4.

Mandelson P. Le coton et I'OMC. Discours à Bamako (Mali) de Peter Mandelson, Commissaire européen pour le Commerce. 2005. www.europarl.europa.eu/intcoop/acp/60_09/ pdf/speech_mandelson_fr.pdf [consulté le 7/05/2009]. 